\title{
INTRODUCTION TO LIQUID CRYSTALS
}

\author{
* Sunanda D
}

\section{Introduction}

In general, matter is broadly divided into three groups, Solids, Liquids and Gases. When solid is heated it first melts into liquid and on further heating moves to gaseous state. Some materials exhibit interesting intermediate state between solid and liquid called liquid crystals. Liquid crystals (LCs) are defined as "condensed fluid phases with spontaneous anisotropy". The simultaneous possession of liquid like fluidity and solid like molecular order character in a single phase is the unique character of liquid crystals, it gives rise to so many interesting properties exploited by scientists and engineers in the application field. These interesting liquid crystalline materials have been observed for over a century but were not recognized as such until 1880s. In 1888, Friedrich Reinitzer gave first systematic report of the phenomena and reported his observations when he prepared cholesteryl benzoate. This seminal paper appeared in Vol.9. (1888) Of Monatshefte fur chemie (Reinizer 1888) and a translation into English has been published in liquid crystals (Reinitzer 1989) to celebrate the centenary of the paper.

Liquid Crysials are also known as the delicate phase of the matter. The molecules in all LC phases diffuse about much like the molecules of a liquid, but as they do so, they maintain some degree of orientational order and sometimes even positional order. The amount of order in a LC phase is quite small relative to a crystal. In this phase the unique axes of the molecules remain, on average, parallel to each other * Department of Physics, Smt. V.H.D.C.I. of Home Science, Bongalore.
e-mail: d_sunanda@yahoo.com 
leading to a preferred direction in space. It is defined as the director ${ }_{\mathbf{n}}^{\Lambda}$. The order parameter ' $S$ ' is defined to specify the orientational order in a liquid crystal phase,

$$
S=<\frac{3}{2} \cos ^{2} \theta-\frac{1}{2}>
$$

where $\theta$ is the polar angle made by the long axis of the molecule with $\Lambda$, and the angular brackets indicate a statistical average. Order parameter is equal to zero in isotropic liquids and equal to one in perfect crystals. Typical values for the order parameter of a liquid crystal range between 0.3 and 0.9 . The order parameter ' $S$ ' of liquid crystal is among the most important parameters that govern all its physical property. The order parameter of the liquid crystals measured by measuring the macroscopic property like diamagnetism, birefringence, nuclear magnetic resonance and Raman scattering etc.

Thousands of organic compounds form LCs when the solid crystal is heated beyond certain temperature but less than melting point. The mesomorphic (intermediate) phase can be identified visually by its characteristic turbidity or with a polarizing microscope by its optical birefringence and textures. Such LCs may pass through one or more mesophases before it is transformed into the isotropic liquid. LC phase formed due to change in temperature, is called thermotropic, or with the influence of solvents is called lyotropic. The parameters like positional order, orientational order and bond orientational order give the extent to which there is order in liquid crystal sample.

According to the molecular order, thermotropic liquid crystals are classified broadly into three types, following the nomenclature proposed originally by Friedel.

\section{Nematic Liquid Crystal Phase}

The nematic phase of rod like LCs is the simplest LC phase. Nematic LCs posses long-range orientational order of the molecules about some direction, i.e. the molecules tend to align parallel to each other, but no long range translational order i.e. no long range correlation of the molecular center of mass position [Figure 1.1]. A unit vector along this axis is known as the director and is denoted by $\hat{n}^{n}$, which is apolar in character. Hence the nematic phase is not ferroelectric. The preferred direction usually varies from point to point in the medium but a uniformly aligned specimen is optically uniaxial, positive and strongly birefringent. The name 'Nematic' comes from the Greek word for thread, since through a polarizing microscope; there are often many dark lines visible in thick film samples. These 
lines are defects and are called disclinations. The nematic LCs are positive uniaxial materials. The mesophase owes its fluidity to the ease with which the molecules slide past one another while still retaining parallelism. It is very easy to deform an aligned nematic liquid crystal by external agents pressure and temperature. Nematics are capable to translate weak external signals (electrical, magnetic, mechanical) into visible optical effects. This property is useful for certain display applications like watches, sensors etc. Nematic phases occur only with molecules, which do not distinguish between right, and left, either each constituent molecule must be identical to its mirror image.

Figure 1.1 Representation of the molecular order in nematic phase

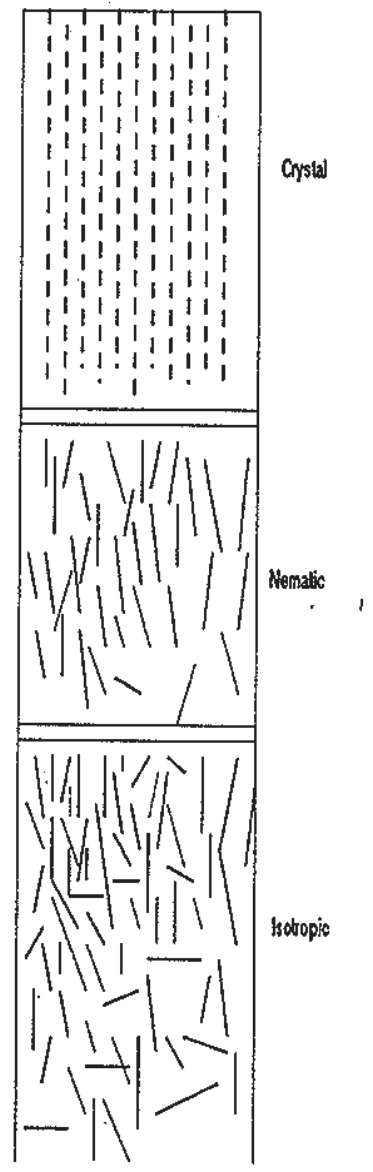




\section{Cholesteric liquid crystal phase}

Nomenclature Cholesteric LCs comes from cholesterol, since many cholesterol esters form these types of liquid crystals. It is a type of nematic liquid crystal that is composed of optically active molecules. The structure can be described as a twisted nematic structure; considering a certain cross sectional plane in the cholesteric phases, the molecules are aligned parallel to a preferred direction $n$ that rotates continuously as one moves along the helix of the phase. The result is a helical structure, the axis of which can be described by a unit vector ${ }_{n}^{\Lambda}$. The twist may be left handed or right handed depending on the molecular conformation. The pitch $\mathrm{P}_{\mathrm{o}}$ of the structure is defined by a distance through which a rotation of $\Lambda$ by $2 p$ along the axis. $P_{0}$ varies widely and falls between $0.2 \mathrm{~mm}$ and macroscopic values. Cholesterics of low pitch, less than about $500 \mathrm{~nm}$, exhibit what are known as blue phases. An infinite pitch corresponds to the nematic structure. The pitch can be adjusted to any desired value by adding appropriate amounts of a chiralic compound to a nematic

Figure 1.2 Schematic representation of the helical structure in cholesteric phase.

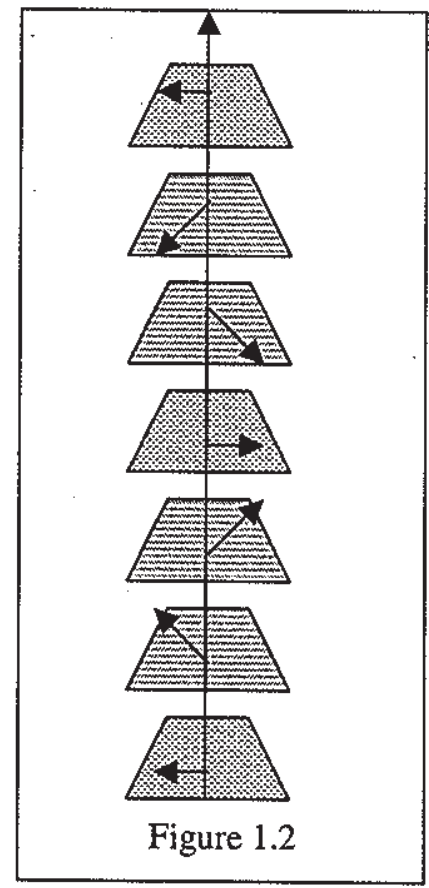


LC. However, the necessary condition for the formation of the cholesteric mesophase is that the molecules are chiralic (optically active) and that is why cholesteric phase is also defined as the chiral nematic phase. Thus, the nematic and cholesteric are sub-classes of the same family. Figure 1.2 shows the equilibrium structure of the cholesteric phase.

\section{Smectic Liquid Crystal Phase}

Smectic is the name coined by $G$. Friedel for certain mesophases with mechanical properties reminiscent of soaps. Smectics are layered structures, with a well-defined interlayer spacing, which can be measured by $X$-ray diffraction. Smectics are thus more ordered than nematics. For a given material, the smectic phases always occur at temperatures below the nematic domain. Smectic liquid crystals have stratified structures but varieties of molecular arrangements are possible within each stratification. Later distinct smectic polymorphs have been identified $S_{A} S_{B}, S_{C}$, $S_{D}, S_{E}, S_{F}, S_{G}, S_{H}, S_{1}, S_{J}, S_{X}, S_{1}$. The letters $A, B$ and $C$ define the three main types of the smectic phases.

In Smectic A the molecules are upright in each layer with their centers irregularly spaced in a 'liquid-like' fashion [Figure 1.3 a]. The layers are able to slide over one another relatively easily because of weak lateral forces between molecules.

Figure 1.3 Schematic representation of the molecular arrangement in (a) Smectic A (b) Smectic C.

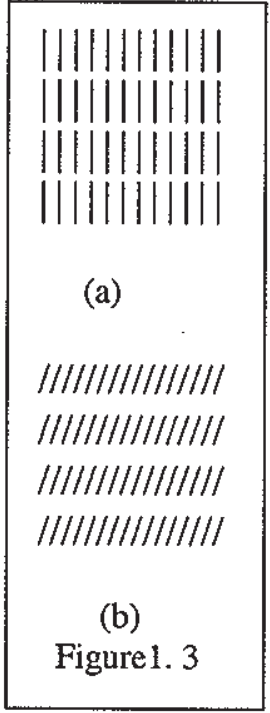


However, if the molecules have strong longitudinal dipole moment, there will be near neighbour antiparallel correlations and this can result in subtle changes in the structure of smectic $A$. Smectic $C$ is a tilted form of smectic $A$, i.e., the molecules are inclined with respect to the layer normal [Figure 1.3 b]. Some of these phases have three dimensional long range positional order as in a crystal, though with weak interlayer forces, while some others referred to as hexatic phases, have three dimensional long range 'bond orientational' order, but without any long range positional order.

\section{Molecules, which form Liquid crystals}

\section{Rod Shaped Molecules}

In rod shaped molecules, molecular axis is much longer than the other two. Such compounds are called calamitic LCs and many different phases are possible with the variation of temperature. It is important that the molecule be rigid for at least some portion of its length, since it must maintain an elongated shape in order to produce interactions that favour alignment. In general structural requirements of the nematic phase, and infact other types of $L C$ phases can be shown by general template as shown in Figure 1.4 for a single compound calamitic LCs. It shows both nematic as well as smectic phases. A liquid crystalline compound does not necessarily have to melt through all the stages of liquid crystalline phases and then to the isotropic liquid, e.g. some materials may melt directly to the nematic phase, whereas others might melt through the smectic phases to the nematic and then

Figure 1.4 A general structural template for generating single compound calamitic LCs

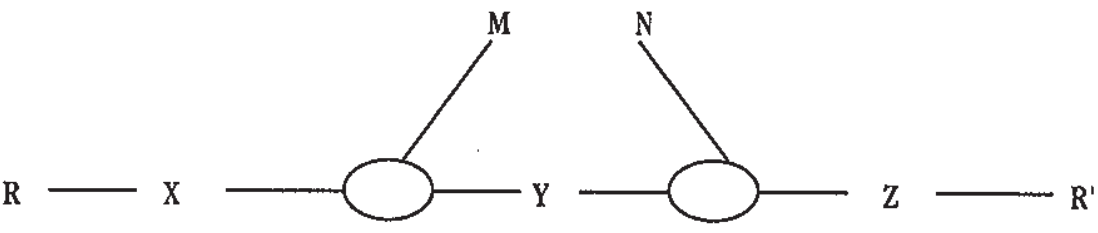

nematic to the amorphous liquid. When chiral molecules are introduced into these liquid crystalline phases, a variety of new phases are formed that have very unusual properties. 


\section{Disc-Shaped Molecules}

It was originally thought that the molecules having rod-like structure could only generate mesomorphism. However later on in 1970, the existence of mesophase generated by disc-like molecules was predicted theoretically. In 1977, Chandrasekhar et al. reported the thermotropic mesomorphism in disc-shaped molecules (hexa-alkanoyl benzenes), namely molecules with one molecular axis much shorter than the other two, also form LC phases. Such compounds are called discotic LCs and again rigidity in the central part of the molecule is essential. The core of a typical discotic LC molecule is usually based on benzene, triphenylene or truxene with 6 or 8 chains, each resembling a typical calamitic LC molecule. Figure 1.5 shows a general structural template of discotic LCs. Structurally, most of them fall into two distinct categories, the columnar and nematic. In columnar phase discs are stacked one on top of the other aperiodically to form liquid like columns, the different columns constituting a two dimensional lattice. A number of variants of this structure have been identified: hexagonal, rectangular, tilted etc. The nematic phase has an orientationally ordered arrangement of the disc without any long-range translational order. A cholesteric phase has also been identified. In recent years significant amount of research has been performed with disc like molecules

Figure 1.5 A general structural template for discotic liquid crystals

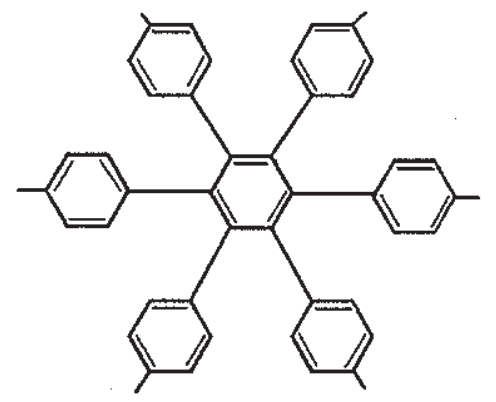

\section{Banana-Shaped Molecules}

Niori et al observed that achiral compounds composed of banana-shaped molecules exhibit ferroelectric phase. New smectic modifications occur in the compounds having this molecular architecture, which have no counterpart in the field of calamitic liquid crystals. A number of such compounds have been synthesized and so far, six different mesophases have been clearly identified. 


\section{Identification of liquid crystal phases}

Several analytical techniques and a great deal of experience requires in the identification of the different liquid crystalline phases and new liquid crystal phases continue to be discovered as the identification techniques improve. The various liquid crystalline phases and other mesophases are characterized and then classified according to the molecular ordering that constitutes the phase structure. All liquid crystalline phases are of importance from a purely academic point of view but some liquid crystalline phases are of particular importance because of their technological applications. Some times different techniques are required employ in the identification of liquid crystalline phase. Optical polarizing microscopy is most widely used technique of liquid crystalline phase identification.

Differential scanning calorimetry is another technique employed to detect the liquid crystal phases. The change in enthalpy associated with a phase transitions does measured. This measurement gives some information about the degree of molecular ordering within a mesophase but cannot identify the type of liquid crystal.

$X$-ray analysis of a liquid crystal will map the positions of the molecules within the phase and hence determine the phase structure and classification to which the particular phase belongs.

Other most simple and commonly used techniques for the identification of mesophases are nuclear magnetic resonance studies, neutron scattering studies.

\section{Properties of liquid crystals}

\section{Mechanical properties}

Liquid crystals are anisotropic fluids, various mechanical properties like viscosity, elasticity may not be same in all directions. In the isotropic liquid phase, there is only one value for the viscosity. Such is not the case for the liquid crystal. If the viscosity is measured by sliding one plate over another, the magnitude of the viscous force depends on whether the director is perpendicular to the plates, parallel to the plates but perpendicular to the motion, or parallel to the plates and parallel to the motion. In each case a different viscosity is measured.

\section{Electromagnetic properties}

The anisotropy of a liquid crystal also affects its electromagnetic properties. The electric and magnetic susceptibilities are different along the director and 
perpendicular to the director. This anisotropy in the susceptibilities is very important, and is responsible for the director of a liquid crystal orienting parallel or perpendicular to an applied field, depending on which susceptibility is larger.

\section{Optical properties}

The anisotropy in the dielectric properties of a liquid crystal is responsible for the linear birefringence. The phenomenon in which the two polarizations have different indices of refraction (ordinary $n_{\circ}$ and extraordinary $n_{e}$ ) and therefore travel along two different directions inside the material is called birefringence or double refraction. The anisotropy of nematic LCs causes light polarized along the director to propagate at a different velocity than light polarized perpendicular to the director. Nematic LCs are therefore birefringent. This fact is never more obvious when a LC is placed between crossed polarizers. However the structure of cholesteric LCs which has a helical structure and the layered structure of smectic LCs affect the propagation of light in ways quite different from what occurs in nematic LCs. In chiral nematic and in chiral smectic LCs circular birefringence takes place.

Figure. 1.6 Representation of the banana shaped molecules.

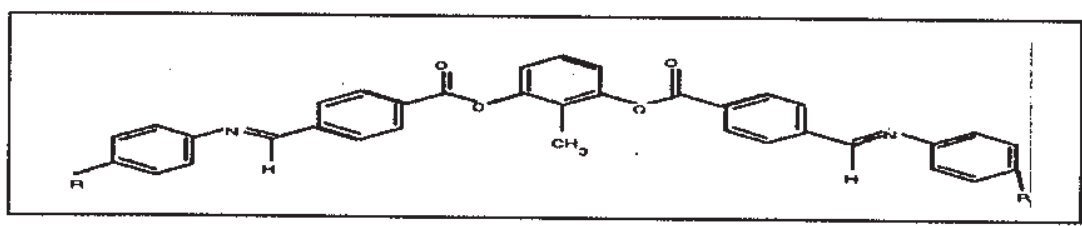

\section{Applications}

Liquid crystals have got a wide range of scientific applications. Nematic and Cholesteric liquid crystals have already found wide applications in display devices. Physical properties like birefringence, optical activity etc., are sensitive to weak external perturbations. The remarkable electro-optic effects have rendered it possible to prepare liquid crystal displays whose main advantage over the other types is that they do not require emission of light and so consume very low power.

Although liquid crystalline materials have been known for over 100 years, largescale applications of the materials in the form of electronic displays did not occur until the mid 1970. When compact, attractive calculators and watches with liquid crystal display (LCD) reached market and soon became household items. The applications of liquid crystal display are rapidly advancing well beyond the early simple watches and calculators to a new level of sophistication, which is far more demanding and therefore is forcing rapid advances in the technology. Nowadays liquid crystals are used in optical computing, nonlinear optics, decorative and 
visual arts, spectroscopy, chromatography and others indicating that there is a relatively broad potential for this technology in future.

Recently ferri-electric liquid crystals have been used for a new generation of fast versatile liquid crystal devices. Polymer dispersed liquid crystals form a relatively new class of materials, which are used in many types of displays, switchable windows and other light shutter devices.

Another area where liquid crystals have made a commercial impact is the field of temperature sensors.

\section{References}

1. Chandrasekhar S., Liquid Crystals, (Cambridge University press: Cambridge) (1994).

2. Satyen Kumar, Liquid Crystals, (Cambridge University press: Cambridge) 2001.

3. Shri Singh, Liquid Crystals, World Scientific Publishing Co.Pte.Ltd. Singapore.

4. Bahadur B. (Editor) Liquid Crystals, Applications and Uses, World Scientific, Vol. I and II (1991).

5. de Gennes P.G., The physics of liquid crystal (UK: Oxford Univ. Press), (1974).

6. Gray G.W., Molecular structure and the properties of Liquid crystals (Academic Press, London and New York), (1962).

7. Gray G.W., and Winsor P.A., Liquid crystals and Plastic Crystals (Ellie Honwood, Chichnester), Vol II (1974).

8. Peter J. Collings and Michael Hird, Introduction to liquid crystals, (Taylor \& Francis Ltd) (1997)

9. Peter J. Collings and Jay S. Potel, Handbook of Liquid crystal research, (Oxford University Press, New York) (1997). 